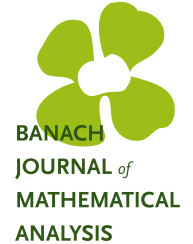

Banach J. Math. Anal. 10 (2016), no. 3, 547-565

http://dx.doi.org/10.1215/17358787-3607288

ISSN: $1735-8787$ (electronic)

http://projecteuclid.org/bjma

ANALYSIS

\title{
LINEAR MAPS BETWEEN C*-ALGEBRAS PRESERVING EXTREME POINTS AND STRONGLY LINEAR PRESERVERS
}

\author{
MARÍA J. BURGOS, ${ }^{1}$ ANTONIO C. MÁRQUEZ-GARCÍA, ${ }^{2}$ \\ ANTONIO MORALES-CAMPOY, ${ }^{2}$ and ANTONIO M. PERALTA ${ }^{3 *}$ \\ To the memory of Professor Wilhelm Kaup \\ Communicated by N.-C. Wong
}

\begin{abstract}
We study new classes of linear preservers between $\mathrm{C}^{*}$-algebras and between $\mathrm{JB}^{*}$-triples. Let $E$ and $F$ be $\mathrm{JB}^{*}$-triples with $\partial_{e}\left(E_{1}\right) \neq \emptyset$. We prove that every linear map $T: E \rightarrow F$ strongly preserving Brown-Pedersen quasi-invertible elements is a triple homomorphism. Among the consequences, we establish that, given two unital $\mathrm{C}^{*}$-algebras $A$ and $B$, for each linear map $T$ strongly preserving Brown-Pedersen quasi-invertible elements, there exists a Jordan *-homomorphism $S: A \rightarrow B$ satisfying $T(x)=T(1) S(x)$ for every $x \in A$. We also study the connections between linear maps strongly preserving Brown-Pedersen quasi-invertibility and other clases of linear preservers between $\mathrm{C}^{*}$-algebras like Bergmann-zero pairs preservers, Brown-Pedersen quasiinvertibility preservers, and extreme points preservers.
\end{abstract}

\section{IntRoduCtion}

Let $X$ be a Banach space. In many favorable cases, the set $\partial_{e}\left(X_{1}\right)$, of all extreme points of the closed unit ball, $X_{1}$, of $X$, reveals many of the geometric properties of the whole Banach space $X$. There are spaces $X$ with $\partial_{e}\left(X_{1}\right)=\emptyset$; however, the Krein-Milman theorem guarantees that $\partial_{e}\left(X_{1}\right)$ is nonempty when $X$ is a dual space.

Copyright 2016 by the Tusi Mathematical Research Group.

Received Jul. 22, 2015; Accepted Nov. 11, 2015.

${ }^{*}$ Corresponding author.

2010 Mathematics Subject Classification. Primary 47B49; Secondary 15A09, 46L05, 47B48.

Keywords. $\mathrm{C}^{*}$-algebra, $\mathrm{JB}^{*}$-triple, triple homomorphism, linear preservers, extreme points preserver, strongly Brown-Pedersen quasi-invertibility preserver. 


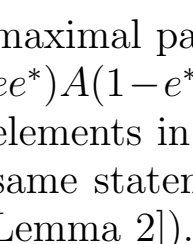

Banach J. Math. Anal. 10 (2016), no. 3, 547-565

http://dx.doi.org/10.1215/17358787-3607288

ISSN: $1735-8787$ (electronic)

http://projecteuclid.org/bjma

ANALYSIS

\title{
LINEAR MAPS BETWEEN C*-ALGEBRAS PRESERVING EXTREME POINTS AND STRONGLY LINEAR PRESERVERS
}

\author{
MARÍA J. BURGOS, ${ }^{1}$ ANTONIO C. MÁRQUEZ-GARCÍA, ${ }^{2}$ \\ ANTONIO MORALES-CAMPOY, ${ }^{2}$ and ANTONIO M. PERALTA ${ }^{3 *}$ \\ To the memory of Professor Wilhelm Kaup \\ Communicated by N.-C. Wong
}

\begin{abstract}
We study new classes of linear preservers between $\mathrm{C}^{*}$-algebras and between $\mathrm{JB}^{*}$-triples. Let $E$ and $F$ be $\mathrm{JB}^{*}$-triples with $\partial_{e}\left(E_{1}\right) \neq \emptyset$. We prove that every linear map $T: E \rightarrow F$ strongly preserving Brown-Pedersen quasi-invertible elements is a triple homomorphism. Among the consequences, we establish that, given two unital $\mathrm{C}^{*}$-algebras $A$ and $B$, for each linear map $T$ strongly preserving Brown-Pedersen quasi-invertible elements, there exists a Jordan *-homomorphism $S: A \rightarrow B$ satisfying $T(x)=T(1) S(x)$ for every $x \in A$. We also study the connections between linear maps strongly preserving Brown-Pedersen quasi-invertibility and other clases of linear preservers between $\mathrm{C}^{*}$-algebras like Bergmann-zero pairs preservers, Brown-Pedersen quasiinvertibility preservers, and extreme points preservers.
\end{abstract}

\section{IntRoduCtion}

Let $X$ be a Banach space. In many favorable cases, the set $\partial_{e}\left(X_{1}\right)$, of all extreme points of the closed unit ball, $X_{1}$, of $X$, reveals many of the geometric properties of the whole Banach space $X$. There are spaces $X$ with $\partial_{e}\left(X_{1}\right)=\emptyset$; however, the Krein-Milman theorem guarantees that $\partial_{e}\left(X_{1}\right)$ is nonempty when $X$ is a dual space.

Copyright 2016 by the Tusi Mathematical Research Group.

Received Jul. 22, 2015; Accepted Nov. 11, 2015.

${ }^{*}$ Corresponding author.

2010 Mathematics Subject Classification. Primary 47B49; Secondary 15A09, 46L05, 47B48.

Keywords. $\mathrm{C}^{*}$-algebra, $\mathrm{JB}^{*}$-triple, triple homomorphism, linear preservers, extreme points preserver, strongly Brown-Pedersen quasi-invertibility preserver. 


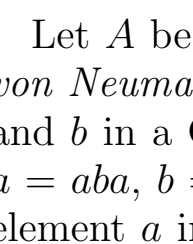

Banach J. Math. Anal. 10 (2016), no. 3, 547-565

http://dx.doi.org/10.1215/17358787-3607288

ISSN: $1735-8787$ (electronic)

http://projecteuclid.org/bjma

ANALYSIS

\title{
LINEAR MAPS BETWEEN C*-ALGEBRAS PRESERVING EXTREME POINTS AND STRONGLY LINEAR PRESERVERS
}

\author{
MARÍA J. BURGOS, ${ }^{1}$ ANTONIO C. MÁRQUEZ-GARCÍA, ${ }^{2}$ \\ ANTONIO MORALES-CAMPOY, ${ }^{2}$ and ANTONIO M. PERALTA ${ }^{3 *}$ \\ To the memory of Professor Wilhelm Kaup \\ Communicated by N.-C. Wong
}

\begin{abstract}
We study new classes of linear preservers between $\mathrm{C}^{*}$-algebras and between $\mathrm{JB}^{*}$-triples. Let $E$ and $F$ be $\mathrm{JB}^{*}$-triples with $\partial_{e}\left(E_{1}\right) \neq \emptyset$. We prove that every linear map $T: E \rightarrow F$ strongly preserving Brown-Pedersen quasi-invertible elements is a triple homomorphism. Among the consequences, we establish that, given two unital $\mathrm{C}^{*}$-algebras $A$ and $B$, for each linear map $T$ strongly preserving Brown-Pedersen quasi-invertible elements, there exists a Jordan *-homomorphism $S: A \rightarrow B$ satisfying $T(x)=T(1) S(x)$ for every $x \in A$. We also study the connections between linear maps strongly preserving Brown-Pedersen quasi-invertibility and other clases of linear preservers between $\mathrm{C}^{*}$-algebras like Bergmann-zero pairs preservers, Brown-Pedersen quasiinvertibility preservers, and extreme points preservers.
\end{abstract}

\section{IntRoduCtion}

Let $X$ be a Banach space. In many favorable cases, the set $\partial_{e}\left(X_{1}\right)$, of all extreme points of the closed unit ball, $X_{1}$, of $X$, reveals many of the geometric properties of the whole Banach space $X$. There are spaces $X$ with $\partial_{e}\left(X_{1}\right)=\emptyset$; however, the Krein-Milman theorem guarantees that $\partial_{e}\left(X_{1}\right)$ is nonempty when $X$ is a dual space.

Copyright 2016 by the Tusi Mathematical Research Group.

Received Jul. 22, 2015; Accepted Nov. 11, 2015.

${ }^{*}$ Corresponding author.

2010 Mathematics Subject Classification. Primary 47B49; Secondary 15A09, 46L05, 47B48.

Keywords. $\mathrm{C}^{*}$-algebra, $\mathrm{JB}^{*}$-triple, triple homomorphism, linear preservers, extreme points preserver, strongly Brown-Pedersen quasi-invertibility preserver. 


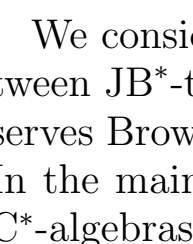

Banach J. Math. Anal. 10 (2016), no. 3, 547-565

http://dx.doi.org/10.1215/17358787-3607288

ISSN: $1735-8787$ (electronic)

http://projecteuclid.org/bjma

ANALYSIS

\title{
LINEAR MAPS BETWEEN C*-ALGEBRAS PRESERVING EXTREME POINTS AND STRONGLY LINEAR PRESERVERS
}

\author{
MARÍA J. BURGOS, ${ }^{1}$ ANTONIO C. MÁRQUEZ-GARCÍA, ${ }^{2}$ \\ ANTONIO MORALES-CAMPOY, ${ }^{2}$ and ANTONIO M. PERALTA ${ }^{3 *}$ \\ To the memory of Professor Wilhelm Kaup \\ Communicated by N.-C. Wong
}

\begin{abstract}
We study new classes of linear preservers between $\mathrm{C}^{*}$-algebras and between $\mathrm{JB}^{*}$-triples. Let $E$ and $F$ be $\mathrm{JB}^{*}$-triples with $\partial_{e}\left(E_{1}\right) \neq \emptyset$. We prove that every linear map $T: E \rightarrow F$ strongly preserving Brown-Pedersen quasi-invertible elements is a triple homomorphism. Among the consequences, we establish that, given two unital $\mathrm{C}^{*}$-algebras $A$ and $B$, for each linear map $T$ strongly preserving Brown-Pedersen quasi-invertible elements, there exists a Jordan *-homomorphism $S: A \rightarrow B$ satisfying $T(x)=T(1) S(x)$ for every $x \in A$. We also study the connections between linear maps strongly preserving Brown-Pedersen quasi-invertibility and other clases of linear preservers between $\mathrm{C}^{*}$-algebras like Bergmann-zero pairs preservers, Brown-Pedersen quasiinvertibility preservers, and extreme points preservers.
\end{abstract}

\section{IntRoduCtion}

Let $X$ be a Banach space. In many favorable cases, the set $\partial_{e}\left(X_{1}\right)$, of all extreme points of the closed unit ball, $X_{1}$, of $X$, reveals many of the geometric properties of the whole Banach space $X$. There are spaces $X$ with $\partial_{e}\left(X_{1}\right)=\emptyset$; however, the Krein-Milman theorem guarantees that $\partial_{e}\left(X_{1}\right)$ is nonempty when $X$ is a dual space.

Copyright 2016 by the Tusi Mathematical Research Group.

Received Jul. 22, 2015; Accepted Nov. 11, 2015.

${ }^{*}$ Corresponding author.

2010 Mathematics Subject Classification. Primary 47B49; Secondary 15A09, 46L05, 47B48.

Keywords. $\mathrm{C}^{*}$-algebra, $\mathrm{JB}^{*}$-triple, triple homomorphism, linear preservers, extreme points preserver, strongly Brown-Pedersen quasi-invertibility preserver. 


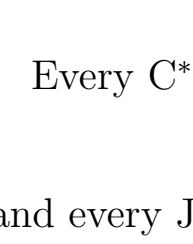

Banach J. Math. Anal. 10 (2016), no. 3, 547-565

http://dx.doi.org/10.1215/17358787-3607288

ISSN: $1735-8787$ (electronic)

http://projecteuclid.org/bjma

ANALYSIS

\title{
LINEAR MAPS BETWEEN C*-ALGEBRAS PRESERVING EXTREME POINTS AND STRONGLY LINEAR PRESERVERS
}

\author{
MARÍA J. BURGOS, ${ }^{1}$ ANTONIO C. MÁRQUEZ-GARCÍA, ${ }^{2}$ \\ ANTONIO MORALES-CAMPOY, ${ }^{2}$ and ANTONIO M. PERALTA ${ }^{3 *}$ \\ To the memory of Professor Wilhelm Kaup \\ Communicated by N.-C. Wong
}

\begin{abstract}
We study new classes of linear preservers between $\mathrm{C}^{*}$-algebras and between $\mathrm{JB}^{*}$-triples. Let $E$ and $F$ be $\mathrm{JB}^{*}$-triples with $\partial_{e}\left(E_{1}\right) \neq \emptyset$. We prove that every linear map $T: E \rightarrow F$ strongly preserving Brown-Pedersen quasi-invertible elements is a triple homomorphism. Among the consequences, we establish that, given two unital $\mathrm{C}^{*}$-algebras $A$ and $B$, for each linear map $T$ strongly preserving Brown-Pedersen quasi-invertible elements, there exists a Jordan *-homomorphism $S: A \rightarrow B$ satisfying $T(x)=T(1) S(x)$ for every $x \in A$. We also study the connections between linear maps strongly preserving Brown-Pedersen quasi-invertibility and other clases of linear preservers between $\mathrm{C}^{*}$-algebras like Bergmann-zero pairs preservers, Brown-Pedersen quasiinvertibility preservers, and extreme points preservers.
\end{abstract}

\section{IntRoduCtion}

Let $X$ be a Banach space. In many favorable cases, the set $\partial_{e}\left(X_{1}\right)$, of all extreme points of the closed unit ball, $X_{1}$, of $X$, reveals many of the geometric properties of the whole Banach space $X$. There are spaces $X$ with $\partial_{e}\left(X_{1}\right)=\emptyset$; however, the Krein-Milman theorem guarantees that $\partial_{e}\left(X_{1}\right)$ is nonempty when $X$ is a dual space.

Copyright 2016 by the Tusi Mathematical Research Group.

Received Jul. 22, 2015; Accepted Nov. 11, 2015.

${ }^{*}$ Corresponding author.

2010 Mathematics Subject Classification. Primary 47B49; Secondary 15A09, 46L05, 47B48.

Keywords. $\mathrm{C}^{*}$-algebra, $\mathrm{JB}^{*}$-triple, triple homomorphism, linear preservers, extreme points preserver, strongly Brown-Pedersen quasi-invertibility preserver. 


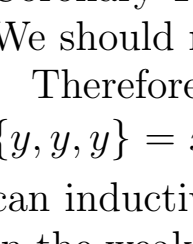

Banach J. Math. Anal. 10 (2016), no. 3, 547-565

http://dx.doi.org/10.1215/17358787-3607288

ISSN: $1735-8787$ (electronic)

http://projecteuclid.org/bjma

ANALYSIS

\title{
LINEAR MAPS BETWEEN C*-ALGEBRAS PRESERVING EXTREME POINTS AND STRONGLY LINEAR PRESERVERS
}

\author{
MARÍA J. BURGOS, ${ }^{1}$ ANTONIO C. MÁRQUEZ-GARCÍA, ${ }^{2}$ \\ ANTONIO MORALES-CAMPOY, ${ }^{2}$ and ANTONIO M. PERALTA ${ }^{3 *}$ \\ To the memory of Professor Wilhelm Kaup \\ Communicated by N.-C. Wong
}

\begin{abstract}
We study new classes of linear preservers between $\mathrm{C}^{*}$-algebras and between $\mathrm{JB}^{*}$-triples. Let $E$ and $F$ be $\mathrm{JB}^{*}$-triples with $\partial_{e}\left(E_{1}\right) \neq \emptyset$. We prove that every linear map $T: E \rightarrow F$ strongly preserving Brown-Pedersen quasi-invertible elements is a triple homomorphism. Among the consequences, we establish that, given two unital $\mathrm{C}^{*}$-algebras $A$ and $B$, for each linear map $T$ strongly preserving Brown-Pedersen quasi-invertible elements, there exists a Jordan *-homomorphism $S: A \rightarrow B$ satisfying $T(x)=T(1) S(x)$ for every $x \in A$. We also study the connections between linear maps strongly preserving Brown-Pedersen quasi-invertibility and other clases of linear preservers between $\mathrm{C}^{*}$-algebras like Bergmann-zero pairs preservers, Brown-Pedersen quasiinvertibility preservers, and extreme points preservers.
\end{abstract}

\section{IntRoduCtion}

Let $X$ be a Banach space. In many favorable cases, the set $\partial_{e}\left(X_{1}\right)$, of all extreme points of the closed unit ball, $X_{1}$, of $X$, reveals many of the geometric properties of the whole Banach space $X$. There are spaces $X$ with $\partial_{e}\left(X_{1}\right)=\emptyset$; however, the Krein-Milman theorem guarantees that $\partial_{e}\left(X_{1}\right)$ is nonempty when $X$ is a dual space.

Copyright 2016 by the Tusi Mathematical Research Group.

Received Jul. 22, 2015; Accepted Nov. 11, 2015.

${ }^{*}$ Corresponding author.

2010 Mathematics Subject Classification. Primary 47B49; Secondary 15A09, 46L05, 47B48.

Keywords. $\mathrm{C}^{*}$-algebra, $\mathrm{JB}^{*}$-triple, triple homomorphism, linear preservers, extreme points preserver, strongly Brown-Pedersen quasi-invertibility preserver. 


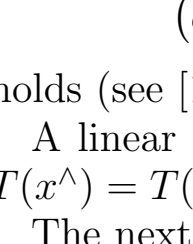

Banach J. Math. Anal. 10 (2016), no. 3, 547-565

http://dx.doi.org/10.1215/17358787-3607288

ISSN: $1735-8787$ (electronic)

http://projecteuclid.org/bjma

ANALYSIS

\title{
LINEAR MAPS BETWEEN C*-ALGEBRAS PRESERVING EXTREME POINTS AND STRONGLY LINEAR PRESERVERS
}

\author{
MARÍA J. BURGOS, ${ }^{1}$ ANTONIO C. MÁRQUEZ-GARCÍA, ${ }^{2}$ \\ ANTONIO MORALES-CAMPOY, ${ }^{2}$ and ANTONIO M. PERALTA ${ }^{3 *}$ \\ To the memory of Professor Wilhelm Kaup \\ Communicated by N.-C. Wong
}

\begin{abstract}
We study new classes of linear preservers between $\mathrm{C}^{*}$-algebras and between $\mathrm{JB}^{*}$-triples. Let $E$ and $F$ be $\mathrm{JB}^{*}$-triples with $\partial_{e}\left(E_{1}\right) \neq \emptyset$. We prove that every linear map $T: E \rightarrow F$ strongly preserving Brown-Pedersen quasi-invertible elements is a triple homomorphism. Among the consequences, we establish that, given two unital $\mathrm{C}^{*}$-algebras $A$ and $B$, for each linear map $T$ strongly preserving Brown-Pedersen quasi-invertible elements, there exists a Jordan *-homomorphism $S: A \rightarrow B$ satisfying $T(x)=T(1) S(x)$ for every $x \in A$. We also study the connections between linear maps strongly preserving Brown-Pedersen quasi-invertibility and other clases of linear preservers between $\mathrm{C}^{*}$-algebras like Bergmann-zero pairs preservers, Brown-Pedersen quasiinvertibility preservers, and extreme points preservers.
\end{abstract}

\section{IntRoduCtion}

Let $X$ be a Banach space. In many favorable cases, the set $\partial_{e}\left(X_{1}\right)$, of all extreme points of the closed unit ball, $X_{1}$, of $X$, reveals many of the geometric properties of the whole Banach space $X$. There are spaces $X$ with $\partial_{e}\left(X_{1}\right)=\emptyset$; however, the Krein-Milman theorem guarantees that $\partial_{e}\left(X_{1}\right)$ is nonempty when $X$ is a dual space.

Copyright 2016 by the Tusi Mathematical Research Group.

Received Jul. 22, 2015; Accepted Nov. 11, 2015.

${ }^{*}$ Corresponding author.

2010 Mathematics Subject Classification. Primary 47B49; Secondary 15A09, 46L05, 47B48.

Keywords. $\mathrm{C}^{*}$-algebra, $\mathrm{JB}^{*}$-triple, triple homomorphism, linear preservers, extreme points preserver, strongly Brown-Pedersen quasi-invertibility preserver. 


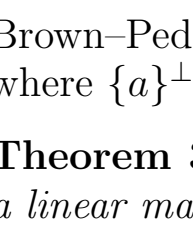

Banach J. Math. Anal. 10 (2016), no. 3, 547-565

http://dx.doi.org/10.1215/17358787-3607288

ISSN: $1735-8787$ (electronic)

http://projecteuclid.org/bjma

ANALYSIS

\title{
LINEAR MAPS BETWEEN C*-ALGEBRAS PRESERVING EXTREME POINTS AND STRONGLY LINEAR PRESERVERS
}

\author{
MARÍA J. BURGOS, ${ }^{1}$ ANTONIO C. MÁRQUEZ-GARCÍA, ${ }^{2}$ \\ ANTONIO MORALES-CAMPOY, ${ }^{2}$ and ANTONIO M. PERALTA ${ }^{3 *}$ \\ To the memory of Professor Wilhelm Kaup \\ Communicated by N.-C. Wong
}

\begin{abstract}
We study new classes of linear preservers between $\mathrm{C}^{*}$-algebras and between $\mathrm{JB}^{*}$-triples. Let $E$ and $F$ be $\mathrm{JB}^{*}$-triples with $\partial_{e}\left(E_{1}\right) \neq \emptyset$. We prove that every linear map $T: E \rightarrow F$ strongly preserving Brown-Pedersen quasi-invertible elements is a triple homomorphism. Among the consequences, we establish that, given two unital $\mathrm{C}^{*}$-algebras $A$ and $B$, for each linear map $T$ strongly preserving Brown-Pedersen quasi-invertible elements, there exists a Jordan *-homomorphism $S: A \rightarrow B$ satisfying $T(x)=T(1) S(x)$ for every $x \in A$. We also study the connections between linear maps strongly preserving Brown-Pedersen quasi-invertibility and other clases of linear preservers between $\mathrm{C}^{*}$-algebras like Bergmann-zero pairs preservers, Brown-Pedersen quasiinvertibility preservers, and extreme points preservers.
\end{abstract}

\section{IntRoduCtion}

Let $X$ be a Banach space. In many favorable cases, the set $\partial_{e}\left(X_{1}\right)$, of all extreme points of the closed unit ball, $X_{1}$, of $X$, reveals many of the geometric properties of the whole Banach space $X$. There are spaces $X$ with $\partial_{e}\left(X_{1}\right)=\emptyset$; however, the Krein-Milman theorem guarantees that $\partial_{e}\left(X_{1}\right)$ is nonempty when $X$ is a dual space.

Copyright 2016 by the Tusi Mathematical Research Group.

Received Jul. 22, 2015; Accepted Nov. 11, 2015.

${ }^{*}$ Corresponding author.

2010 Mathematics Subject Classification. Primary 47B49; Secondary 15A09, 46L05, 47B48.

Keywords. $\mathrm{C}^{*}$-algebra, $\mathrm{JB}^{*}$-triple, triple homomorphism, linear preservers, extreme points preserver, strongly Brown-Pedersen quasi-invertibility preserver. 


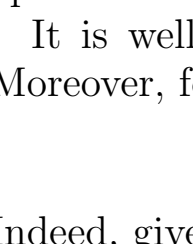

Banach J. Math. Anal. 10 (2016), no. 3, 547-565

http://dx.doi.org/10.1215/17358787-3607288

ISSN: $1735-8787$ (electronic)

http://projecteuclid.org/bjma

ANALYSIS

\title{
LINEAR MAPS BETWEEN C*-ALGEBRAS PRESERVING EXTREME POINTS AND STRONGLY LINEAR PRESERVERS
}

\author{
MARÍA J. BURGOS, ${ }^{1}$ ANTONIO C. MÁRQUEZ-GARCÍA, ${ }^{2}$ \\ ANTONIO MORALES-CAMPOY, ${ }^{2}$ and ANTONIO M. PERALTA ${ }^{3 *}$ \\ To the memory of Professor Wilhelm Kaup \\ Communicated by N.-C. Wong
}

\begin{abstract}
We study new classes of linear preservers between $\mathrm{C}^{*}$-algebras and between $\mathrm{JB}^{*}$-triples. Let $E$ and $F$ be $\mathrm{JB}^{*}$-triples with $\partial_{e}\left(E_{1}\right) \neq \emptyset$. We prove that every linear map $T: E \rightarrow F$ strongly preserving Brown-Pedersen quasi-invertible elements is a triple homomorphism. Among the consequences, we establish that, given two unital $\mathrm{C}^{*}$-algebras $A$ and $B$, for each linear map $T$ strongly preserving Brown-Pedersen quasi-invertible elements, there exists a Jordan *-homomorphism $S: A \rightarrow B$ satisfying $T(x)=T(1) S(x)$ for every $x \in A$. We also study the connections between linear maps strongly preserving Brown-Pedersen quasi-invertibility and other clases of linear preservers between $\mathrm{C}^{*}$-algebras like Bergmann-zero pairs preservers, Brown-Pedersen quasiinvertibility preservers, and extreme points preservers.
\end{abstract}

\section{IntRoduCtion}

Let $X$ be a Banach space. In many favorable cases, the set $\partial_{e}\left(X_{1}\right)$, of all extreme points of the closed unit ball, $X_{1}$, of $X$, reveals many of the geometric properties of the whole Banach space $X$. There are spaces $X$ with $\partial_{e}\left(X_{1}\right)=\emptyset$; however, the Krein-Milman theorem guarantees that $\partial_{e}\left(X_{1}\right)$ is nonempty when $X$ is a dual space.

Copyright 2016 by the Tusi Mathematical Research Group.

Received Jul. 22, 2015; Accepted Nov. 11, 2015.

${ }^{*}$ Corresponding author.

2010 Mathematics Subject Classification. Primary 47B49; Secondary 15A09, 46L05, 47B48.

Keywords. $\mathrm{C}^{*}$-algebra, $\mathrm{JB}^{*}$-triple, triple homomorphism, linear preservers, extreme points preserver, strongly Brown-Pedersen quasi-invertibility preserver. 


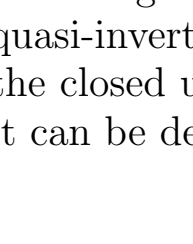

Banach J. Math. Anal. 10 (2016), no. 3, 547-565

http://dx.doi.org/10.1215/17358787-3607288

ISSN: $1735-8787$ (electronic)

http://projecteuclid.org/bjma

ANALYSIS

\title{
LINEAR MAPS BETWEEN C*-ALGEBRAS PRESERVING EXTREME POINTS AND STRONGLY LINEAR PRESERVERS
}

\author{
MARÍA J. BURGOS, ${ }^{1}$ ANTONIO C. MÁRQUEZ-GARCÍA, ${ }^{2}$ \\ ANTONIO MORALES-CAMPOY, ${ }^{2}$ and ANTONIO M. PERALTA ${ }^{3 *}$ \\ To the memory of Professor Wilhelm Kaup \\ Communicated by N.-C. Wong
}

\begin{abstract}
We study new classes of linear preservers between $\mathrm{C}^{*}$-algebras and between $\mathrm{JB}^{*}$-triples. Let $E$ and $F$ be $\mathrm{JB}^{*}$-triples with $\partial_{e}\left(E_{1}\right) \neq \emptyset$. We prove that every linear map $T: E \rightarrow F$ strongly preserving Brown-Pedersen quasi-invertible elements is a triple homomorphism. Among the consequences, we establish that, given two unital $\mathrm{C}^{*}$-algebras $A$ and $B$, for each linear map $T$ strongly preserving Brown-Pedersen quasi-invertible elements, there exists a Jordan *-homomorphism $S: A \rightarrow B$ satisfying $T(x)=T(1) S(x)$ for every $x \in A$. We also study the connections between linear maps strongly preserving Brown-Pedersen quasi-invertibility and other clases of linear preservers between $\mathrm{C}^{*}$-algebras like Bergmann-zero pairs preservers, Brown-Pedersen quasiinvertibility preservers, and extreme points preservers.
\end{abstract}

\section{IntRoduCtion}

Let $X$ be a Banach space. In many favorable cases, the set $\partial_{e}\left(X_{1}\right)$, of all extreme points of the closed unit ball, $X_{1}$, of $X$, reveals many of the geometric properties of the whole Banach space $X$. There are spaces $X$ with $\partial_{e}\left(X_{1}\right)=\emptyset$; however, the Krein-Milman theorem guarantees that $\partial_{e}\left(X_{1}\right)$ is nonempty when $X$ is a dual space.

Copyright 2016 by the Tusi Mathematical Research Group.

Received Jul. 22, 2015; Accepted Nov. 11, 2015.

${ }^{*}$ Corresponding author.

2010 Mathematics Subject Classification. Primary 47B49; Secondary 15A09, 46L05, 47B48.

Keywords. $\mathrm{C}^{*}$-algebra, $\mathrm{JB}^{*}$-triple, triple homomorphism, linear preservers, extreme points preserver, strongly Brown-Pedersen quasi-invertibility preserver. 


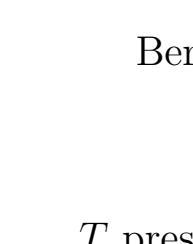

Banach J. Math. Anal. 10 (2016), no. 3, 547-565

http://dx.doi.org/10.1215/17358787-3607288

ISSN: $1735-8787$ (electronic)

http://projecteuclid.org/bjma

ANALYSIS

\title{
LINEAR MAPS BETWEEN C*-ALGEBRAS PRESERVING EXTREME POINTS AND STRONGLY LINEAR PRESERVERS
}

\author{
MARÍA J. BURGOS, ${ }^{1}$ ANTONIO C. MÁRQUEZ-GARCÍA, ${ }^{2}$ \\ ANTONIO MORALES-CAMPOY, ${ }^{2}$ and ANTONIO M. PERALTA ${ }^{3 *}$ \\ To the memory of Professor Wilhelm Kaup \\ Communicated by N.-C. Wong
}

\begin{abstract}
We study new classes of linear preservers between $\mathrm{C}^{*}$-algebras and between $\mathrm{JB}^{*}$-triples. Let $E$ and $F$ be $\mathrm{JB}^{*}$-triples with $\partial_{e}\left(E_{1}\right) \neq \emptyset$. We prove that every linear map $T: E \rightarrow F$ strongly preserving Brown-Pedersen quasi-invertible elements is a triple homomorphism. Among the consequences, we establish that, given two unital $\mathrm{C}^{*}$-algebras $A$ and $B$, for each linear map $T$ strongly preserving Brown-Pedersen quasi-invertible elements, there exists a Jordan *-homomorphism $S: A \rightarrow B$ satisfying $T(x)=T(1) S(x)$ for every $x \in A$. We also study the connections between linear maps strongly preserving Brown-Pedersen quasi-invertibility and other clases of linear preservers between $\mathrm{C}^{*}$-algebras like Bergmann-zero pairs preservers, Brown-Pedersen quasiinvertibility preservers, and extreme points preservers.
\end{abstract}

\section{IntRoduCtion}

Let $X$ be a Banach space. In many favorable cases, the set $\partial_{e}\left(X_{1}\right)$, of all extreme points of the closed unit ball, $X_{1}$, of $X$, reveals many of the geometric properties of the whole Banach space $X$. There are spaces $X$ with $\partial_{e}\left(X_{1}\right)=\emptyset$; however, the Krein-Milman theorem guarantees that $\partial_{e}\left(X_{1}\right)$ is nonempty when $X$ is a dual space.

Copyright 2016 by the Tusi Mathematical Research Group.

Received Jul. 22, 2015; Accepted Nov. 11, 2015.

${ }^{*}$ Corresponding author.

2010 Mathematics Subject Classification. Primary 47B49; Secondary 15A09, 46L05, 47B48.

Keywords. $\mathrm{C}^{*}$-algebra, $\mathrm{JB}^{*}$-triple, triple homomorphism, linear preservers, extreme points preserver, strongly Brown-Pedersen quasi-invertibility preserver. 


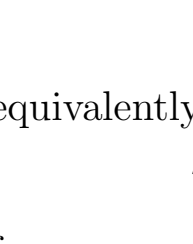

Banach J. Math. Anal. 10 (2016), no. 3, 547-565

http://dx.doi.org/10.1215/17358787-3607288

ISSN: $1735-8787$ (electronic)

http://projecteuclid.org/bjma

ANALYSIS

\title{
LINEAR MAPS BETWEEN C*-ALGEBRAS PRESERVING EXTREME POINTS AND STRONGLY LINEAR PRESERVERS
}

\author{
MARÍA J. BURGOS, ${ }^{1}$ ANTONIO C. MÁRQUEZ-GARCÍA, ${ }^{2}$ \\ ANTONIO MORALES-CAMPOY, ${ }^{2}$ and ANTONIO M. PERALTA ${ }^{3 *}$ \\ To the memory of Professor Wilhelm Kaup \\ Communicated by N.-C. Wong
}

\begin{abstract}
We study new classes of linear preservers between $\mathrm{C}^{*}$-algebras and between $\mathrm{JB}^{*}$-triples. Let $E$ and $F$ be $\mathrm{JB}^{*}$-triples with $\partial_{e}\left(E_{1}\right) \neq \emptyset$. We prove that every linear map $T: E \rightarrow F$ strongly preserving Brown-Pedersen quasi-invertible elements is a triple homomorphism. Among the consequences, we establish that, given two unital $\mathrm{C}^{*}$-algebras $A$ and $B$, for each linear map $T$ strongly preserving Brown-Pedersen quasi-invertible elements, there exists a Jordan *-homomorphism $S: A \rightarrow B$ satisfying $T(x)=T(1) S(x)$ for every $x \in A$. We also study the connections between linear maps strongly preserving Brown-Pedersen quasi-invertibility and other clases of linear preservers between $\mathrm{C}^{*}$-algebras like Bergmann-zero pairs preservers, Brown-Pedersen quasiinvertibility preservers, and extreme points preservers.
\end{abstract}

\section{IntRoduCtion}

Let $X$ be a Banach space. In many favorable cases, the set $\partial_{e}\left(X_{1}\right)$, of all extreme points of the closed unit ball, $X_{1}$, of $X$, reveals many of the geometric properties of the whole Banach space $X$. There are spaces $X$ with $\partial_{e}\left(X_{1}\right)=\emptyset$; however, the Krein-Milman theorem guarantees that $\partial_{e}\left(X_{1}\right)$ is nonempty when $X$ is a dual space.

Copyright 2016 by the Tusi Mathematical Research Group.

Received Jul. 22, 2015; Accepted Nov. 11, 2015.

${ }^{*}$ Corresponding author.

2010 Mathematics Subject Classification. Primary 47B49; Secondary 15A09, 46L05, 47B48.

Keywords. $\mathrm{C}^{*}$-algebra, $\mathrm{JB}^{*}$-triple, triple homomorphism, linear preservers, extreme points preserver, strongly Brown-Pedersen quasi-invertibility preserver. 


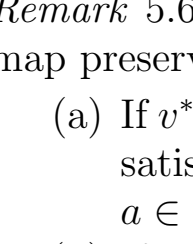

Banach J. Math. Anal. 10 (2016), no. 3, 547-565

http://dx.doi.org/10.1215/17358787-3607288

ISSN: $1735-8787$ (electronic)

http://projecteuclid.org/bjma

ANALYSIS

\title{
LINEAR MAPS BETWEEN C*-ALGEBRAS PRESERVING EXTREME POINTS AND STRONGLY LINEAR PRESERVERS
}

\author{
MARÍA J. BURGOS, ${ }^{1}$ ANTONIO C. MÁRQUEZ-GARCÍA, ${ }^{2}$ \\ ANTONIO MORALES-CAMPOY, ${ }^{2}$ and ANTONIO M. PERALTA ${ }^{3 *}$ \\ To the memory of Professor Wilhelm Kaup \\ Communicated by N.-C. Wong
}

\begin{abstract}
We study new classes of linear preservers between $\mathrm{C}^{*}$-algebras and between $\mathrm{JB}^{*}$-triples. Let $E$ and $F$ be $\mathrm{JB}^{*}$-triples with $\partial_{e}\left(E_{1}\right) \neq \emptyset$. We prove that every linear map $T: E \rightarrow F$ strongly preserving Brown-Pedersen quasi-invertible elements is a triple homomorphism. Among the consequences, we establish that, given two unital $\mathrm{C}^{*}$-algebras $A$ and $B$, for each linear map $T$ strongly preserving Brown-Pedersen quasi-invertible elements, there exists a Jordan *-homomorphism $S: A \rightarrow B$ satisfying $T(x)=T(1) S(x)$ for every $x \in A$. We also study the connections between linear maps strongly preserving Brown-Pedersen quasi-invertibility and other clases of linear preservers between $\mathrm{C}^{*}$-algebras like Bergmann-zero pairs preservers, Brown-Pedersen quasiinvertibility preservers, and extreme points preservers.
\end{abstract}

\section{IntRoduCtion}

Let $X$ be a Banach space. In many favorable cases, the set $\partial_{e}\left(X_{1}\right)$, of all extreme points of the closed unit ball, $X_{1}$, of $X$, reveals many of the geometric properties of the whole Banach space $X$. There are spaces $X$ with $\partial_{e}\left(X_{1}\right)=\emptyset$; however, the Krein-Milman theorem guarantees that $\partial_{e}\left(X_{1}\right)$ is nonempty when $X$ is a dual space.

Copyright 2016 by the Tusi Mathematical Research Group.

Received Jul. 22, 2015; Accepted Nov. 11, 2015.

${ }^{*}$ Corresponding author.

2010 Mathematics Subject Classification. Primary 47B49; Secondary 15A09, 46L05, 47B48.

Keywords. $\mathrm{C}^{*}$-algebra, $\mathrm{JB}^{*}$-triple, triple homomorphism, linear preservers, extreme points preserver, strongly Brown-Pedersen quasi-invertibility preserver. 


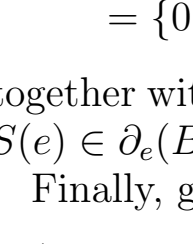

Banach J. Math. Anal. 10 (2016), no. 3, 547-565

http://dx.doi.org/10.1215/17358787-3607288

ISSN: $1735-8787$ (electronic)

http://projecteuclid.org/bjma

ANALYSIS

\title{
LINEAR MAPS BETWEEN C*-ALGEBRAS PRESERVING EXTREME POINTS AND STRONGLY LINEAR PRESERVERS
}

\author{
MARÍA J. BURGOS, ${ }^{1}$ ANTONIO C. MÁRQUEZ-GARCÍA, ${ }^{2}$ \\ ANTONIO MORALES-CAMPOY, ${ }^{2}$ and ANTONIO M. PERALTA ${ }^{3 *}$ \\ To the memory of Professor Wilhelm Kaup \\ Communicated by N.-C. Wong
}

\begin{abstract}
We study new classes of linear preservers between $\mathrm{C}^{*}$-algebras and between $\mathrm{JB}^{*}$-triples. Let $E$ and $F$ be $\mathrm{JB}^{*}$-triples with $\partial_{e}\left(E_{1}\right) \neq \emptyset$. We prove that every linear map $T: E \rightarrow F$ strongly preserving Brown-Pedersen quasi-invertible elements is a triple homomorphism. Among the consequences, we establish that, given two unital $\mathrm{C}^{*}$-algebras $A$ and $B$, for each linear map $T$ strongly preserving Brown-Pedersen quasi-invertible elements, there exists a Jordan *-homomorphism $S: A \rightarrow B$ satisfying $T(x)=T(1) S(x)$ for every $x \in A$. We also study the connections between linear maps strongly preserving Brown-Pedersen quasi-invertibility and other clases of linear preservers between $\mathrm{C}^{*}$-algebras like Bergmann-zero pairs preservers, Brown-Pedersen quasiinvertibility preservers, and extreme points preservers.
\end{abstract}

\section{IntRoduCtion}

Let $X$ be a Banach space. In many favorable cases, the set $\partial_{e}\left(X_{1}\right)$, of all extreme points of the closed unit ball, $X_{1}$, of $X$, reveals many of the geometric properties of the whole Banach space $X$. There are spaces $X$ with $\partial_{e}\left(X_{1}\right)=\emptyset$; however, the Krein-Milman theorem guarantees that $\partial_{e}\left(X_{1}\right)$ is nonempty when $X$ is a dual space.

Copyright 2016 by the Tusi Mathematical Research Group.

Received Jul. 22, 2015; Accepted Nov. 11, 2015.

${ }^{*}$ Corresponding author.

2010 Mathematics Subject Classification. Primary 47B49; Secondary 15A09, 46L05, 47B48.

Keywords. $\mathrm{C}^{*}$-algebra, $\mathrm{JB}^{*}$-triple, triple homomorphism, linear preservers, extreme points preserver, strongly Brown-Pedersen quasi-invertibility preserver. 


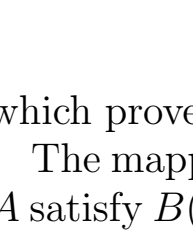

Banach J. Math. Anal. 10 (2016), no. 3, 547-565

http://dx.doi.org/10.1215/17358787-3607288

ISSN: $1735-8787$ (electronic)

http://projecteuclid.org/bjma

ANALYSIS

\title{
LINEAR MAPS BETWEEN C*-ALGEBRAS PRESERVING EXTREME POINTS AND STRONGLY LINEAR PRESERVERS
}

\author{
MARÍA J. BURGOS, ${ }^{1}$ ANTONIO C. MÁRQUEZ-GARCÍA, ${ }^{2}$ \\ ANTONIO MORALES-CAMPOY, ${ }^{2}$ and ANTONIO M. PERALTA ${ }^{3 *}$ \\ To the memory of Professor Wilhelm Kaup \\ Communicated by N.-C. Wong
}

\begin{abstract}
We study new classes of linear preservers between $\mathrm{C}^{*}$-algebras and between $\mathrm{JB}^{*}$-triples. Let $E$ and $F$ be $\mathrm{JB}^{*}$-triples with $\partial_{e}\left(E_{1}\right) \neq \emptyset$. We prove that every linear map $T: E \rightarrow F$ strongly preserving Brown-Pedersen quasi-invertible elements is a triple homomorphism. Among the consequences, we establish that, given two unital $\mathrm{C}^{*}$-algebras $A$ and $B$, for each linear map $T$ strongly preserving Brown-Pedersen quasi-invertible elements, there exists a Jordan *-homomorphism $S: A \rightarrow B$ satisfying $T(x)=T(1) S(x)$ for every $x \in A$. We also study the connections between linear maps strongly preserving Brown-Pedersen quasi-invertibility and other clases of linear preservers between $\mathrm{C}^{*}$-algebras like Bergmann-zero pairs preservers, Brown-Pedersen quasiinvertibility preservers, and extreme points preservers.
\end{abstract}

\section{IntRoduCtion}

Let $X$ be a Banach space. In many favorable cases, the set $\partial_{e}\left(X_{1}\right)$, of all extreme points of the closed unit ball, $X_{1}$, of $X$, reveals many of the geometric properties of the whole Banach space $X$. There are spaces $X$ with $\partial_{e}\left(X_{1}\right)=\emptyset$; however, the Krein-Milman theorem guarantees that $\partial_{e}\left(X_{1}\right)$ is nonempty when $X$ is a dual space.

Copyright 2016 by the Tusi Mathematical Research Group.

Received Jul. 22, 2015; Accepted Nov. 11, 2015.

${ }^{*}$ Corresponding author.

2010 Mathematics Subject Classification. Primary 47B49; Secondary 15A09, 46L05, 47B48.

Keywords. $\mathrm{C}^{*}$-algebra, $\mathrm{JB}^{*}$-triple, triple homomorphism, linear preservers, extreme points preserver, strongly Brown-Pedersen quasi-invertibility preserver. 


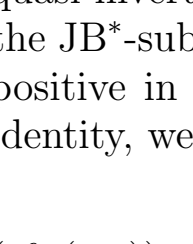

Banach J. Math. Anal. 10 (2016), no. 3, 547-565

http://dx.doi.org/10.1215/17358787-3607288

ISSN: $1735-8787$ (electronic)

http://projecteuclid.org/bjma

ANALYSIS

\title{
LINEAR MAPS BETWEEN C*-ALGEBRAS PRESERVING EXTREME POINTS AND STRONGLY LINEAR PRESERVERS
}

\author{
MARÍA J. BURGOS, ${ }^{1}$ ANTONIO C. MÁRQUEZ-GARCÍA, ${ }^{2}$ \\ ANTONIO MORALES-CAMPOY, ${ }^{2}$ and ANTONIO M. PERALTA ${ }^{3 *}$ \\ To the memory of Professor Wilhelm Kaup \\ Communicated by N.-C. Wong
}

\begin{abstract}
We study new classes of linear preservers between $\mathrm{C}^{*}$-algebras and between $\mathrm{JB}^{*}$-triples. Let $E$ and $F$ be $\mathrm{JB}^{*}$-triples with $\partial_{e}\left(E_{1}\right) \neq \emptyset$. We prove that every linear map $T: E \rightarrow F$ strongly preserving Brown-Pedersen quasi-invertible elements is a triple homomorphism. Among the consequences, we establish that, given two unital $\mathrm{C}^{*}$-algebras $A$ and $B$, for each linear map $T$ strongly preserving Brown-Pedersen quasi-invertible elements, there exists a Jordan *-homomorphism $S: A \rightarrow B$ satisfying $T(x)=T(1) S(x)$ for every $x \in A$. We also study the connections between linear maps strongly preserving Brown-Pedersen quasi-invertibility and other clases of linear preservers between $\mathrm{C}^{*}$-algebras like Bergmann-zero pairs preservers, Brown-Pedersen quasiinvertibility preservers, and extreme points preservers.
\end{abstract}

\section{IntRoduCtion}

Let $X$ be a Banach space. In many favorable cases, the set $\partial_{e}\left(X_{1}\right)$, of all extreme points of the closed unit ball, $X_{1}$, of $X$, reveals many of the geometric properties of the whole Banach space $X$. There are spaces $X$ with $\partial_{e}\left(X_{1}\right)=\emptyset$; however, the Krein-Milman theorem guarantees that $\partial_{e}\left(X_{1}\right)$ is nonempty when $X$ is a dual space.

Copyright 2016 by the Tusi Mathematical Research Group.

Received Jul. 22, 2015; Accepted Nov. 11, 2015.

${ }^{*}$ Corresponding author.

2010 Mathematics Subject Classification. Primary 47B49; Secondary 15A09, 46L05, 47B48.

Keywords. $\mathrm{C}^{*}$-algebra, $\mathrm{JB}^{*}$-triple, triple homomorphism, linear preservers, extreme points preserver, strongly Brown-Pedersen quasi-invertibility preserver. 


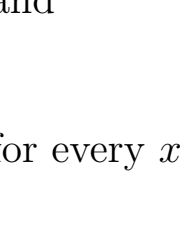

Banach J. Math. Anal. 10 (2016), no. 3, 547-565

http://dx.doi.org/10.1215/17358787-3607288

ISSN: $1735-8787$ (electronic)

http://projecteuclid.org/bjma

ANALYSIS

\title{
LINEAR MAPS BETWEEN C*-ALGEBRAS PRESERVING EXTREME POINTS AND STRONGLY LINEAR PRESERVERS
}

\author{
MARÍA J. BURGOS, ${ }^{1}$ ANTONIO C. MÁRQUEZ-GARCÍA, ${ }^{2}$ \\ ANTONIO MORALES-CAMPOY, ${ }^{2}$ and ANTONIO M. PERALTA ${ }^{3 *}$ \\ To the memory of Professor Wilhelm Kaup \\ Communicated by N.-C. Wong
}

\begin{abstract}
We study new classes of linear preservers between $\mathrm{C}^{*}$-algebras and between $\mathrm{JB}^{*}$-triples. Let $E$ and $F$ be $\mathrm{JB}^{*}$-triples with $\partial_{e}\left(E_{1}\right) \neq \emptyset$. We prove that every linear map $T: E \rightarrow F$ strongly preserving Brown-Pedersen quasi-invertible elements is a triple homomorphism. Among the consequences, we establish that, given two unital $\mathrm{C}^{*}$-algebras $A$ and $B$, for each linear map $T$ strongly preserving Brown-Pedersen quasi-invertible elements, there exists a Jordan *-homomorphism $S: A \rightarrow B$ satisfying $T(x)=T(1) S(x)$ for every $x \in A$. We also study the connections between linear maps strongly preserving Brown-Pedersen quasi-invertibility and other clases of linear preservers between $\mathrm{C}^{*}$-algebras like Bergmann-zero pairs preservers, Brown-Pedersen quasiinvertibility preservers, and extreme points preservers.
\end{abstract}

\section{IntRoduCtion}

Let $X$ be a Banach space. In many favorable cases, the set $\partial_{e}\left(X_{1}\right)$, of all extreme points of the closed unit ball, $X_{1}$, of $X$, reveals many of the geometric properties of the whole Banach space $X$. There are spaces $X$ with $\partial_{e}\left(X_{1}\right)=\emptyset$; however, the Krein-Milman theorem guarantees that $\partial_{e}\left(X_{1}\right)$ is nonempty when $X$ is a dual space.

Copyright 2016 by the Tusi Mathematical Research Group.

Received Jul. 22, 2015; Accepted Nov. 11, 2015.

${ }^{*}$ Corresponding author.

2010 Mathematics Subject Classification. Primary 47B49; Secondary 15A09, 46L05, 47B48.

Keywords. $\mathrm{C}^{*}$-algebra, $\mathrm{JB}^{*}$-triple, triple homomorphism, linear preservers, extreme points preserver, strongly Brown-Pedersen quasi-invertibility preserver. 


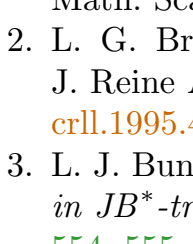

Banach J. Math. Anal. 10 (2016), no. 3, 547-565

http://dx.doi.org/10.1215/17358787-3607288

ISSN: $1735-8787$ (electronic)

http://projecteuclid.org/bjma

ANALYSIS

\title{
LINEAR MAPS BETWEEN C*-ALGEBRAS PRESERVING EXTREME POINTS AND STRONGLY LINEAR PRESERVERS
}

\author{
MARÍA J. BURGOS, ${ }^{1}$ ANTONIO C. MÁRQUEZ-GARCÍA, ${ }^{2}$ \\ ANTONIO MORALES-CAMPOY, ${ }^{2}$ and ANTONIO M. PERALTA ${ }^{3 *}$ \\ To the memory of Professor Wilhelm Kaup \\ Communicated by N.-C. Wong
}

\begin{abstract}
We study new classes of linear preservers between $\mathrm{C}^{*}$-algebras and between $\mathrm{JB}^{*}$-triples. Let $E$ and $F$ be $\mathrm{JB}^{*}$-triples with $\partial_{e}\left(E_{1}\right) \neq \emptyset$. We prove that every linear map $T: E \rightarrow F$ strongly preserving Brown-Pedersen quasi-invertible elements is a triple homomorphism. Among the consequences, we establish that, given two unital $\mathrm{C}^{*}$-algebras $A$ and $B$, for each linear map $T$ strongly preserving Brown-Pedersen quasi-invertible elements, there exists a Jordan *-homomorphism $S: A \rightarrow B$ satisfying $T(x)=T(1) S(x)$ for every $x \in A$. We also study the connections between linear maps strongly preserving Brown-Pedersen quasi-invertibility and other clases of linear preservers between $\mathrm{C}^{*}$-algebras like Bergmann-zero pairs preservers, Brown-Pedersen quasiinvertibility preservers, and extreme points preservers.
\end{abstract}

\section{IntRoduCtion}

Let $X$ be a Banach space. In many favorable cases, the set $\partial_{e}\left(X_{1}\right)$, of all extreme points of the closed unit ball, $X_{1}$, of $X$, reveals many of the geometric properties of the whole Banach space $X$. There are spaces $X$ with $\partial_{e}\left(X_{1}\right)=\emptyset$; however, the Krein-Milman theorem guarantees that $\partial_{e}\left(X_{1}\right)$ is nonempty when $X$ is a dual space.

Copyright 2016 by the Tusi Mathematical Research Group.

Received Jul. 22, 2015; Accepted Nov. 11, 2015.

${ }^{*}$ Corresponding author.

2010 Mathematics Subject Classification. Primary 47B49; Secondary 15A09, 46L05, 47B48.

Keywords. $\mathrm{C}^{*}$-algebra, $\mathrm{JB}^{*}$-triple, triple homomorphism, linear preservers, extreme points preserver, strongly Brown-Pedersen quasi-invertibility preserver. 


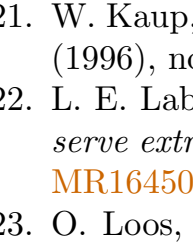

Banach J. Math. Anal. 10 (2016), no. 3, 547-565

http://dx.doi.org/10.1215/17358787-3607288

ISSN: $1735-8787$ (electronic)

http://projecteuclid.org/bjma

ANALYSIS

\title{
LINEAR MAPS BETWEEN C*-ALGEBRAS PRESERVING EXTREME POINTS AND STRONGLY LINEAR PRESERVERS
}

\author{
MARÍA J. BURGOS, ${ }^{1}$ ANTONIO C. MÁRQUEZ-GARCÍA, ${ }^{2}$ \\ ANTONIO MORALES-CAMPOY, ${ }^{2}$ and ANTONIO M. PERALTA ${ }^{3 *}$ \\ To the memory of Professor Wilhelm Kaup \\ Communicated by N.-C. Wong
}

\begin{abstract}
We study new classes of linear preservers between $\mathrm{C}^{*}$-algebras and between $\mathrm{JB}^{*}$-triples. Let $E$ and $F$ be $\mathrm{JB}^{*}$-triples with $\partial_{e}\left(E_{1}\right) \neq \emptyset$. We prove that every linear map $T: E \rightarrow F$ strongly preserving Brown-Pedersen quasi-invertible elements is a triple homomorphism. Among the consequences, we establish that, given two unital $\mathrm{C}^{*}$-algebras $A$ and $B$, for each linear map $T$ strongly preserving Brown-Pedersen quasi-invertible elements, there exists a Jordan *-homomorphism $S: A \rightarrow B$ satisfying $T(x)=T(1) S(x)$ for every $x \in A$. We also study the connections between linear maps strongly preserving Brown-Pedersen quasi-invertibility and other clases of linear preservers between $\mathrm{C}^{*}$-algebras like Bergmann-zero pairs preservers, Brown-Pedersen quasiinvertibility preservers, and extreme points preservers.
\end{abstract}

\section{IntRoduCtion}

Let $X$ be a Banach space. In many favorable cases, the set $\partial_{e}\left(X_{1}\right)$, of all extreme points of the closed unit ball, $X_{1}$, of $X$, reveals many of the geometric properties of the whole Banach space $X$. There are spaces $X$ with $\partial_{e}\left(X_{1}\right)=\emptyset$; however, the Krein-Milman theorem guarantees that $\partial_{e}\left(X_{1}\right)$ is nonempty when $X$ is a dual space.

Copyright 2016 by the Tusi Mathematical Research Group.

Received Jul. 22, 2015; Accepted Nov. 11, 2015.

${ }^{*}$ Corresponding author.

2010 Mathematics Subject Classification. Primary 47B49; Secondary 15A09, 46L05, 47B48.

Keywords. $\mathrm{C}^{*}$-algebra, $\mathrm{JB}^{*}$-triple, triple homomorphism, linear preservers, extreme points preserver, strongly Brown-Pedersen quasi-invertibility preserver. 